\title{
Análisis cinemático del prototipo de robot equino desarrollado para el simulador de prácticas de tiro montado en la Escuela Nacional de Carabineros "Alfonso López Pumarejo"
}

Kinematic analysis of the equine robot prototype developed for the shooting practice simulator mounted in the National School of Carabineros "Alfonso López Pumarejo"

Análise cinemática do protótipo de robô eqüino desenvolvido para o simulador de tiro esportivo montado na Escola Nacional de Carabineros "Alfonso López Pumarejo"

\author{
Javier Raúl Romero Roa* \\ Jairo Rojas Ángel** \\ Jhonson Pardo Morales*** \\ Jaime Eduardo Andrade Ramírez ${ }^{* \star *}$ \\ Escuela Nacional de Carabineros - Colombia
}

Fecha de Recibido: Junio 19 del 2018

Fecha de Aceptación: Septiembre 17 del 2018

Fecha de Publicación: Octubre 01 de 2019

DOI: http://dx.doi.org/10.22335/rlct.v10i4.679

* Ingeniero ambiental, especialista en Gestión ambiental docente investigador, ESCAR-DINAE-Nro.COL0061592 de la Escuela Nacional de Carabineros. Correo electrónico: Javier.raul1268@correo.policia.gov.co Orcid: https://orcid.org/0000-0003-0596-6472

** Técnico profesional en servicio de Policía, Tecnólogo en planeación para el Control Ambiental, docente investigador, Coinvestigador grupo de investigación ESCAR-DINAE-Nro.COL0061592. Correo electrónico: Jairo.rojas7245@correo.policia.gov.co Orcid: https://orcid.org/00000003-2231-4054

*** Técnico profesional en servicio de policía, Coinvestigador del grupo de investigación ESCAR-DINAE -Nro.COL0061592 de la Escuela Nacional de Carabineros Correo electrónico: jhonson.pardo@correo.policia.gov.co.
Orcid: https://orcid.org/0000-0001-7370-2677

****Ingeniero electrónico, Maestría en sistemas automáticos de producción, docente de la universidad de Cundinamarca, integrante del grupo de investigación en software y tecnologías Facatativá "GISTFA" de la Universidad de Cundinamarca, Coinvestigador del grupo de investigación ESCAR-DINAE Nro.COL0061592 de la Escuela Nacional de Carabineros, Correo electrónico: jandrade76@yahoo.com Orcid:

https://orcid.org/0000-0001-6077-2449

\section{Resumen}

Este artículo de investigación comprende describir de forma aproximada el modelamiento cinemático directo del robot equino de seis 
grados de libertad "6-DOF" desarrollado como producto de la investigación en curso titulada "Simulador equino para prácticas de tiro montado en la ESCAR". El manipulador diseñado tiene fines educativos y será utilizado en las prácticas de tiro montado, como herramienta de apoyo didáctico en los cursos de carabineros de la policía nacional. Se presenta la propuesta de diseño del manipulador robótico adaptado a las necesidades educativas. Se utilizan actuadores eléctricos lineales de potencia que acoplan los eslabones de la cadena cinemática propuesta. Se aproxima la cinemática directa del robot, utilizando el algoritmo de Denavit-Hartenberg "DH" y se calcula el modelo dinámico del manipulador. Finalmente se valida el prototipo realizando pruebas de simulación del comportamiento del manipulador mediante la creación de segmentos de programa en Matlab $\mathbb{R}$ y se comparan con los resultados obtenidos mediante el control del mecanismo desde una aplicación de software desarrollada para tal fin en Microsoft Visual C\# 2013®

\section{Palabras clave}

Cinemática Directa, robot equino, DenavitHartenberg, simulador de tiro montado.

\section{Abstract}

This research article includes an approximate description of the direct kinematic modeling of the 6-degree-of-freedom equine robot developed as a product of the ongoing research entitled "Equine simulator for shooting practice mounted on the ESCAR". The designed manipulator has educational purposes and will be used in mounted shooting practices, as a teaching support tool in the national police Carabineros courses. The design proposal of the robotic manipulator adapted to the educational needs is presented. Linear electric power actuators are used that connect the links of the proposed kinematic chain. The direct kinematics of the robot is approximated, using the algorithm of Denavit-Hartenberg "DH" and the dynamic model of the manipulator is calculated. Finally, the prototype is validated by simulating the behavior of the manipulator through the creation

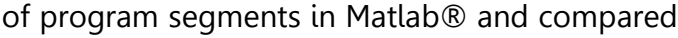
with the results obtained by controlling the mechanism from a software application developed for this purpose in Microsoft Visual C \# 2013®

\section{Keywords}

Direct kinematics, equine robot, DenavitHartenberg. Mounted shooting simulator

\section{Introducción}

En general se mencionan en este artículo las etapas que permitieron la construcción del robot partiendo del requerimiento de movimientos, análisis de movimientos, estudio y selección de actuadores, sistemas de control y potencia, interfaz de control de usuario y finalmente la validación y las pruebas.

En la construcción del prototipo de robot equino se consideró el análisis cinemático como el primer paso para describir el modelo geométrico en forma paramétrica.[1] En ésta construcción al igual que en un modelo industrial se presentaron errores de fabricación en el mecanizado y acople de eslabones y actuadores que generaron discrepancias entre los parámetros de diseño y la estructura física, por lo cual se buscó la identificación de los modelos individuales de la cadena cinemática con el fin de aproximarse a los parámetros reales y así tener un buen rendimiento del prototipo desde el punto de vista de la precisión de posicionamiento [2],[3]

Con el fin establecer un comportamiento acertado del prototipo propuesto que desencadene en la obtención de la información de ubicación de cada una de las partes mecánicas del robot se utilizó la metodología de DenavitHartenberg y se deja el estudio presente como base para estudios posteriores del análisis dinámico de trayectorias y de control [4], [5]

Adicionalmente se consideró como efector final la nariz del modelo (punto de control de rienda sobre un equino real) y a partir de la base (sistema de referencia) obtener la relación entre todos los subsistemas partiendo del eslabón cero hasta el punto central del efector final "TCP" [6]. 
Finalmente para el diseño del prototipo se consideraron los movimientos que describieron cada uno de los pasos básicos del equino para el "TCP", los cuales fueron previamente registrados en etapas anteriores del proyecto y se logró aproximar con un modelo de "6DOF" (figura 1) basado en la dinámica de cuerpos rígidos y en el requerimiento de la región de trabajo del sistema. [7]

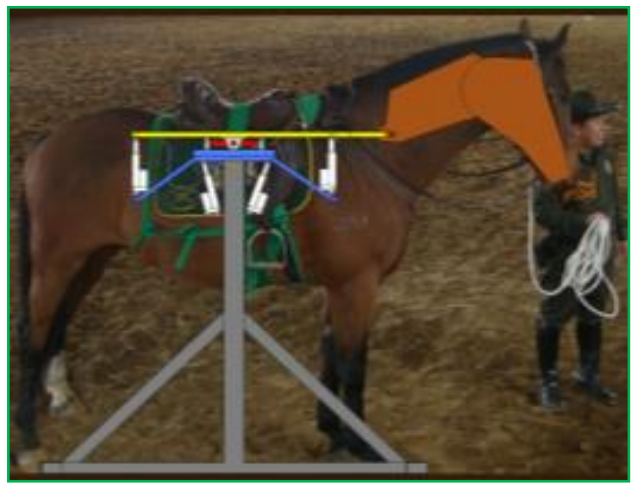

Figura. 1 Diagrama Físico propuesto Fuente: Grupo de investigación UDEC-ESCAR

\section{Metodología}

La metodología seguida en el desarrollo propuesto, se describirá de acuerdo a cada una de las etapas que intervienen en el proceso tal como se muestra en la figura 2, por último se validará el modelo y se presentarán los resultados:

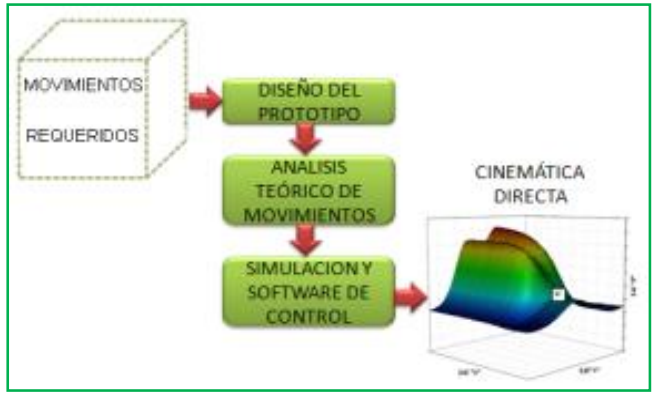

Figura. 2 Diagrama General del proceso Fuente: Grupo de investigación UDEC-ESCAR

\section{Movimientos requeridos:}

Se parte del estudio de los pasos básicos del equino que fueron registrados en fases anteriores del proyecto y dieron como resultado, modelos gráficos que describen los movimientos del caballo desde 13 puntos sobre la estructura ósea equina.

\section{Diseño del prototipo:}

La arquitectura del robot propuesta fue de tipo $6 R$, siendo el efector final el modelo de cabeza del equino y el "TCP" en el punto de (freno o final de rienda). Se consideraron actuadores lineales de potencia y sistemas de control comercialmente programables y adaptables a esta etapa del estudio. Además, Se buscó diseñar un robot sencillo de modelar y de analizar cinemáticamente, sin restricciones relevantes en la multifuncionalidad de los pasos equinos a seguir, lo cual llevó a esta elección inicial, frente a la arquitectura de los robots cuadrúpedos de "n" grados de libertad e inclusive a los bípedos tan extendidos hoy a nivel mundial. [8][9]

\section{Análisis teórico de movimientos:}

En el estudio de la cinemática directa del prototipo en cuestión se hace uso de las herramientas del algebra vectorial y matricial con el fin de aplicar una metodología generalizada y sistemática que describe y representa la localización de los eslabones del robot con respecto a un sistema de coordenadas de referencia. Se considera que todos los elementos mecánicos del sistema giran con respecto al sistema de referencia, por lo tanto se liga un sistema coordenado a cada cuerpo a lo largo de cada eje de la articulación para cada elemento. Seguidamente el problema se reduce a encontrar una matriz de transformación que relaciona el sistema de coordenadas de todos los elementos con los ejes de referencia. Se propone para futuros estudios el uso de herramientas de software especializadas en cinemática directa. [10], [11], [12],[13]

\section{Simulación y software de control:}

En esta parte del trabajo desarrollado se utilizaron herramientas de software de modelado 3D como Sketchup ${ }^{\circledR}$ y freeCad ${ }^{\circledR}$ además de MATLAB® ${ }^{\circledR}$, con el fin de estimar las posiciones y orientaciones relativas de todos los sistemas de coordenadas del manipulador con respecto a los ejes coordenados de referencia y observar el volumen de trabajo del efector final. 
Seguidamente se diseño una interfaz de prueba en Microsoft Visual $C \# \circledast$ que permitiera integrar todo el hardware básico de control para realizar movimientos del robot en forma manual (se introducen los parámetros del robot manipulador propuesto) según las indicaciones del usuario y así poder evaluar cada punto real de posición del "TCP".

Resultados: Se muestran los resultados analíticos y se comparan con los experimentales de tal manera que se comprueben las ecuaciones obtenidas para la cinemática directa en $M A T L A B \otimes$ y concuerden con las posiciones en el espacio del efector final según los seis ángulos ingresados en la interfaz de usuario.

A continuación se mostrará en detalle, el conjunto de métodos, técnicas y herramientas que se emplearon en el proceso de implementación del sistema propuesto.

\section{Movimientos requeridos:}

Como parte inicial de este trabajo se consideró que los registros tomados de 13 sensores inerciales en cada una de las marchas básicas equinas, deberían proporcionar una idea general de los movimientos deseados para el robot. Por lo tanto con toda la información almacenada en etapas previas a este y tomando registros en movimiento (análisis de video en el software Kinovea ${ }^{\circledR}$ ) del "TCP" como marcador sobre el equino real en las diferentes prácticas, se logró cruzar todos estos modelos para obtener curvas aproximadas de los desplazamientos de la cabeza del caballo y referenciarlas con el mínimo de articulaciones posibles para cumplir con el objetivo del estudio. Figura 3.

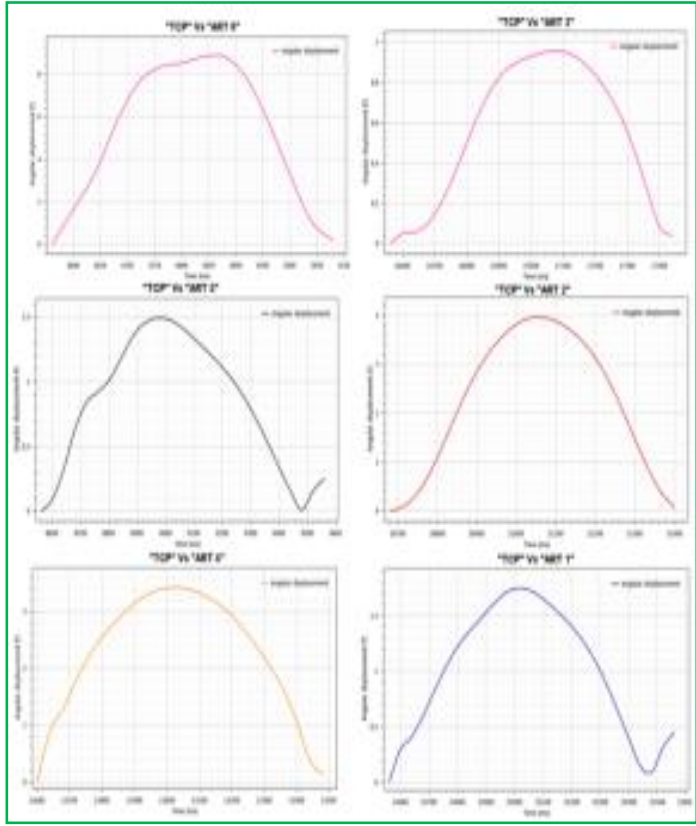

Figura. 3 Desplazamientos angulares del punto central de la herramienta de trabajo "TCP" con relaciona a cada una de las seis articulaciones.

Fuente: Grupo de investigación UDEC-ESCAR

\section{Diseño del prototipo:}

Una vez obtenidas estas curvas de desplazamiento del efector final se logró estimar el modelo gráfico del manipulador y la región de trabajo aproximada [14]. El diseño se aproximó a un manipulador robótico industrial ya que la cinemática de un robot de múltiples puntos de referencia es muy complicada debido a su mecanismo complejo y accionamiento redundante [15]. Se consideró en esta etapa del proyecto que debería cumplir las condiciones mínimas requeridas de posicionamiento espacial, descartando temporalmente parámetros dinámicos; y finalmente adaptarlo para que realice con un mínimo error las marchas básicas equinas.

Como se mencionó anteriormente, el robot es del tipo manipulador y presenta siete eslabones y seis grados de libertad "6 DOF". Cada uno de los grados de libertad es una articulación del tipo rotacional. Tanto los 7 eslabones como las 6 juntas servirán para posicionar espacialmente el extremo del robot. La primera unión, de tipo par 
de revolución, sirve para unir la base del prototipo (eslabón número 0) con el eslabón número 1 y permite el movimiento adelante y atrás de todo el sistema mecánico. La segunda unión de tipo cruceta (2 DOF), une el eslabón número 1 con los eslabones 2 y 3 . Esta plataforma compuesta por los eslabones 2 y 3 describe un par de movimientos rotacionales sobre el plano horizontal Y-Z con desplazamientos del "TCP" de derecha a izquierda, de arriba a abajo y en ambos casos en sentido contrario. El cuarto eslabón está unido a la plataforma mediante una junta rotacional y permite el desplazamiento del cuello hacia arriba y abajo. La unión siguiente de tipo rotacional une al eslabón número 5 y permite girar el cuello a derecha e izquierda. La última junta rotacional une al eslabón 6 (efector final) con toda la cadena cinemática y permite mover la cabeza hacia arriba y abajo. En el extremo del eslabón 6 está el "TCP". Figura (4). Como se tienen 6 juntas rotacionales, esto indica que se tendrán 6 actuadores.

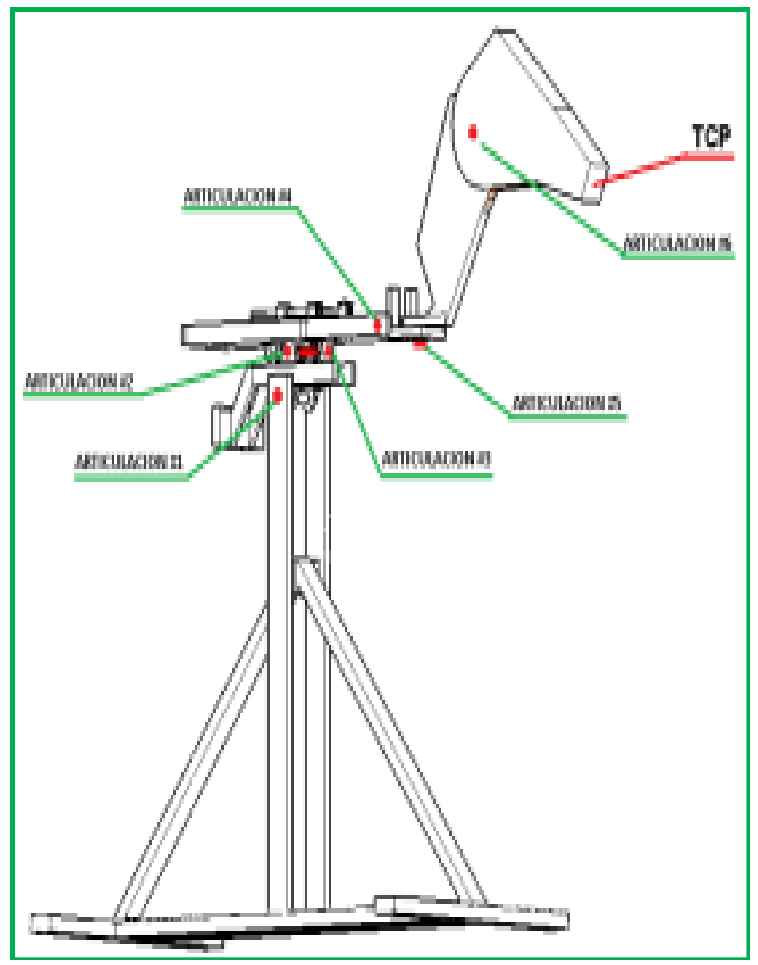

Figura. 4 Diagrama geométrico propuesto Fuente: Grupo de investigación UDEC-ESCAR

\subsubsection{Actuadores}

Los actuadores son los elementos que convierten las señales que vienen del controlador en movimiento de las juntas. Por lo tanto, son los encargados del movimiento del manipulador. Los actuadores más comunes en el campo de la robótica son del tipo eléctrico, neumático e hidráulico y la selección del tipo de actuador a utilizar esta en función de algunas variables como son el tamaño del manipulador, tipo de trabajo a realizar, ambiente de trabajo del manipulador, requerimientos de velocidad y peso a manejar entre otras. De acuerdo a [16] los más utilizados son los de tipo eléctrico. En la Tabla 1 se presentan los pros y contra de los diversos actuadores.

Tabla 1. Ventajas y desventajas de actuadores

\begin{tabular}{|c|c|c|}
\hline $\begin{array}{l}\text { Tipo de } \\
\text { actuador }\end{array}$ & Ventaja & Desventaja \\
\hline Eléctrico & $\begin{array}{l}\text { - Rápidos y } \\
\text { precisos. } \\
\text { - Posibilidad de } \\
\text { aplicar varias } \\
\text { técnicas de } \\
\text { control del } \\
\text { movimiento. } \\
\text { - Más } \\
\text { económicos. } \\
\text { - Tamaño } \\
\text { reducido. } \\
\text { - Tiempos de } \\
\text { respuesta } \\
\text { rápidos. }\end{array}$ & $\begin{array}{l}\text { - Altas velocidades } \\
\text { implican bajo par. } \\
\text { - Necesidad de } \\
\text { engranajes o } \\
\text { transmisiones. } \\
\text { - El juego de los } \\
\text { engranajes limita } \\
\text { la precisión. } \\
\text { - No resultan } \\
\text { adecuados en } \\
\text { atmósferas } \\
\text { inflamables. } \\
\text { - } \\
\text { Sobrecalentamient } \\
\text { o en condiciones } \\
\text { de trabajo de } \\
\text { parada. } \\
\text { - Necesidad de } \\
\text { frenos para } \\
\text { bloquear el } \\
\text { sistema. } \\
\text { - Coste alto en } \\
\text { motores grandes. }\end{array}$ \\
\hline $\begin{array}{l}\text { Neumátic } \\
\text { o }\end{array}$ & $\begin{array}{l}\text { - Más } \\
\text { económicos. } \\
\text { - Alta } \\
\text { velocidad. } \\
\text { - No contamina } \\
\text { el área de } \\
\text { trabajo con } \\
\text { otros fluidos. }\end{array}$ & $\begin{array}{l}\text { - Compresibilidad } \\
\text { del aire limita el } \\
\text { control y la } \\
\text { precisión. } \\
\text { - Mala precisión al } \\
\text { actuar con cargas. }\end{array}$ \\
\hline
\end{tabular}




\begin{tabular}{|c|c|c|}
\hline $\begin{array}{l}\text { Hidráulic } \\
\text { o }\end{array}$ & $\begin{array}{l}\text { - No necesita } \\
\text { línea de } \\
\text { retorno la } \\
\text { instalación. } \\
\text { - Fuente de } \\
\text { energía usual } \\
\text { en entornos } \\
\text { industriales. } \\
\text { - Relación } \\
\text { potencia -peso } \\
\text { muy buena. } \\
\text { - Muy buen } \\
\text { servo control. } \\
\text { - Auto } \\
\text { lubricación y } \\
\text { autorrefrigerad } \\
\text { o. } \\
\text { - Trabajo en } \\
\text { parada sin } \\
\text { problemas. } \\
\text { - Respuesta } \\
\text { rápida. } \\
\text { - Operación } \\
\text { suave a bajas } \\
\text { velocidades. } \\
\text { - Adecuado en } \\
\text { atmósferas } \\
\text { Inflamables. }\end{array}$ & $\begin{array}{l}\text { - Instalación } \\
\text { hidráulica costosa. } \\
\text { - Necesidad de } \\
\text { mantenimiento y } \\
\text { fugas de aceite. } \\
\text { - Necesidad de } \\
\text { línea de retorno } \\
\text { en la instalación. } \\
\text { - Problemas de } \\
\text { miniaturización. }\end{array}$ \\
\hline
\end{tabular}

Fuente: Autores

Según las características de los actuadores, se decidió utilizar del tipo eléctrico y después de varios ensayos se seleccionaron actuadores que además de todas las características mencionadas, tuvieran cajas reductoras, finalmente se optó por dejar los que se muestran en la tabla 2

Tabla 2. Actuadores seleccionados.

\section{Junta Actuador}

Motor lineal Nominal Stroke,12/24

DC, Duty Cycle: 25\%, Protection: IP65,

1, 2, Potentiometer, carga max. $720 \mathrm{Nw}$,

3,4 vel. Sin carga $10 \mathrm{~mm} / \mathrm{seg}$, vel con carga $5 \mathrm{~mm} / \mathrm{seg}$.

Servo Motor ASSY, TORXIS 1.0, hasta $3200 \mathrm{Oz}^{*}$ in de torque, vel. 60 grados en $500 \mathrm{~ms}$, control pwm o entrada de $\mathbf{5 , 6}$ control analógica, control de velocidad y poisicon, 270 grados de recorrido, 12VDC, 3A, peso $2.2 \mathrm{lbm}$, Dimensiones (inches): $5.5 \times 3.9 \times 2.4$.

\begin{abstract}
Cuentan internamente con una tarjeta de control JRK12v3 la cual ya viene pre configurada de fábrica con los parámetros necesarios para su correcto funcionamiento
\end{abstract}

\subsubsection{Partes y dimensiones}

De forma general se presentan en las figuras 5 al 8 las partes que se diseñaron para el robot, empezando desde el eslabón número 0 hasta el efector final, considerando que la selección de los materiales seleccionados pasaron por una serie de pruebas físicas en especial de resistencia, carga y estrés. Además debían cumplir con ser ligeros de peso, ya que se debía trasladar fácilmente el robot de un lugar a otro y tener dimensiones similares a las de los equinos objeto del estudio.

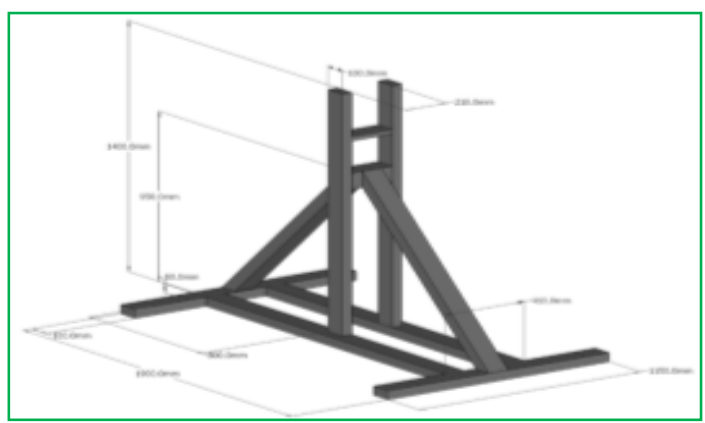

Figura. 5 Eslabón \#0 Base fija del prototipo Fuente: Grupo de investigación UDEC-ESCAR

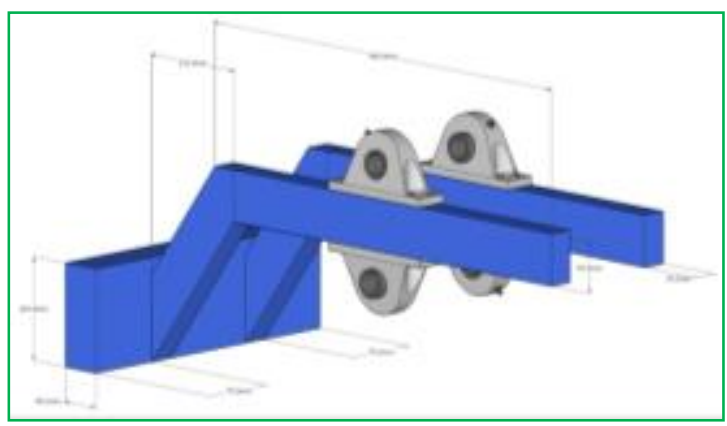

Figura. 6 Eslabón \#1 Base Lomo del prototipo Fuente: Grupo de investigación UDEC-ESCAR 


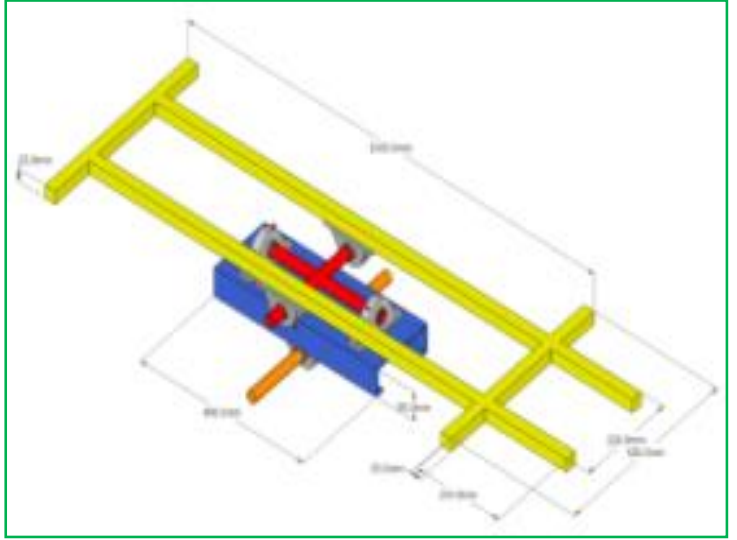

Figura. 7 Eslabón \#2,3 Lomo del prototipo Fuente: Grupo de investigación UDEC-ESCAR

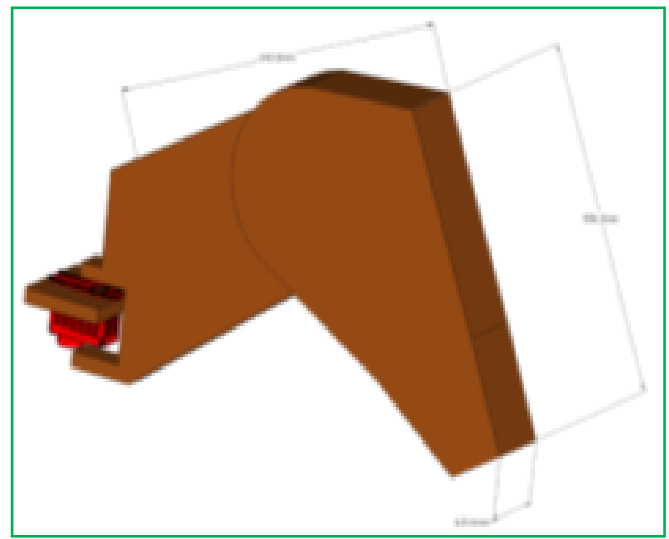

Figura. 8 Eslabón \#4, 5,6 cuello y cabeza del prototipo Fuente: Grupo de investigación UDEC-ESCAR

Una vez diseñadas todas las partes del sistema, se acoplan mediante las diferentes juntas y sistemas de potencia para terminar con el ensamble del prototipo desde CAD hasta la implementación física. Figura 9.

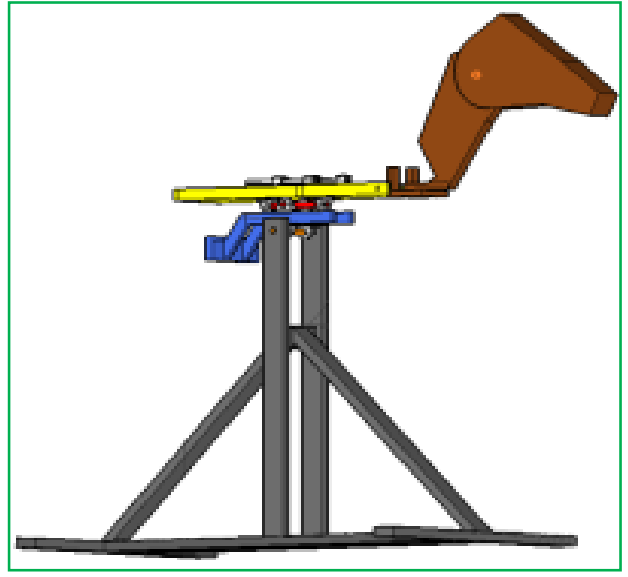

Figura. 9 Modelo CAD del prototipo Fuente: Grupo de investigación UDEC-ESCAR

En la tabla 3 se presentan las características básicas del robot.

Tabla 3. Características básicas del manipulador.

\begin{tabular}{lllllll}
\multicolumn{1}{c}{ ART. } & $\mathbf{1}$ & $\mathbf{2}$ & $\mathbf{3}$ & $\mathbf{4}$ & $\mathbf{5}$ & $\mathbf{6}$ \\
$\begin{array}{l}\text { AMPLITU } \\
\text { D EN }\end{array}$ & 9 & 20 & 5 & 18 & 8 & 43 \\
(RAD) & & & & & & \\
RANGO & 0,1 & 0. & 0,00 & 0.3 & 0.1 & 0.7 \\
DE & $5 /$ & $3 /$ & 8 & $1 /$ & $3 /$ & $5 /$ \\
TRABAJO & 70 & 49 & 72 & 41 & 96 & 04 \\
$\left({ }^{\circ}\right)$ & & & & & & \\
\hline
\end{tabular}

\subsection{Análisis teórico de movimientos Cinemática directa:}

La cinemática directa estudia el movimiento del robot respecto a un sistema de referencia fijo. Consiste en determinar cuál es la posición y orientación del extremo del robot dados los valores de las articulaciones y parámetros geométricos de los elementos del robot en forma analítica ó utilizando otras herramientas matemáticas y de software. [17], [18], [19]

La metodología matricial sistemática de DenavitHartenberg permite la obtención del modelo cinemático directo que define sistemas de coordenadas ligados a cada eslabón del mecanismo determinando la cinemática completa del mismo [20]. Una vez que se han 
definido los sistemas de coordenadas, (Figura 10) es posible pasar de uno a otro a través de una serie de transformaciones básicas que dependen exclusivamente de las características geométricas de cada eslabón; pudiendo así relacionar un sistema de coordenadas con otro. Las transformaciones que se llevan a cabo son:

Rotación alrededor del eje Zi-1 un ángulo "өi". Traslación a lo largo de Zi-1 una distancia "di" ; vector $(0,0, \mathrm{di})$.

Traslación a lo largo de Xi una distancia "ai" ; vector (ai, 0,0).

Rotación alrededor del eje Xi un ángulo" $\boldsymbol{\alpha i " . ~}$

donde:

$\theta i$ Es el ángulo que forman los ejes $\mathrm{Xi}-1$ y $\mathrm{Xi}$ medido en un plano perpendicular al eje Zi-1, utilizando la regla de la mano derecha. Es un parámetro para articulaciones giratorias.

di Es la distancia a lo largo del eje $\mathrm{Zi}-1$ desde el origen del sistema de coordenadas (i-1)-ésimo hasta la intersección del eje Zi-1 con el eje Xi. En articulaciones prismáticas es un parámetro variable.

ai Es la distancia a lo largo del eje Xi que va desde la intersección del eje $\mathrm{Zi}-1$ con el eje Xi hasta el origen del sistema iésimo, en el caso de articulaciones giratorias. En el caso de articulaciones prismáticas, se calcula como la distancia más corta entre los ejes Z1-i y Zi. ai Es el ángulo de separación del eje $\mathrm{Zi}-1$ y el eje $\mathrm{Z} i$, medido en un plano perpendicular al eje $\mathrm{Xi}$, utilizando la regla de la mano derecha. [20].

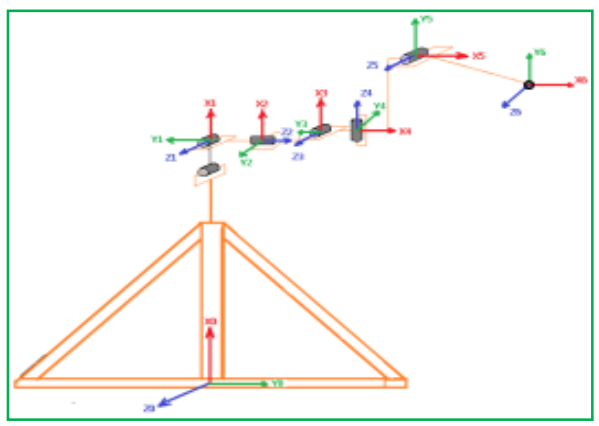

Figura. 10 Sistema Coordenado seleccionado Fuente: Grupo de investigación UDEC-ESCAR

Se presentan como resultado de la metodología usada, los parámetros $\mathrm{DH}$ que se presentan en la tabla 4 para el prototipo desarrollado.
Tabla 4. Parámetros DH del manipulador

$\begin{array}{ccccccc}\text { ART. } & \text { Oi } & \text { di } & \text { ai } & \alpha i & \begin{array}{c}\text { Var. de las } \\ \text { artic. "q" }\end{array} \\ 1 & \text { q1 } & 0 & 153,6 & 0 & \theta 1 \\ 2 & \text { q2 } & 0 & 0 & 90 & \theta 2 \\ 3 & \text { q3 } & 28,6 & 28,6 & 90 & \theta 3 \\ 4 & q 4 & 0 & 10 & 90 & \theta 4 \\ 5 & \text { q5 } & 80 & 80 & 90 & \theta 5 \\ 6 & \text { q6 } & 0 & 58,6 & 0 & \theta 6\end{array}$

Al tener los parámetros de Denavit- Hartenberg se determinan las matrices de transformación homogénea $A_{i}^{i-1}$ para cada sistema coordenado. Esta matriz es un arreglo de $4 \times 4$ que presenta información relativa a la posición y orientación del sistema de referencia unido al i-ésimo enlace del manipulador en relación con el sistema con el sistema de referencia i-1. Por tal motivo, la matriz $A_{1}^{0}$ representa la posición y orientación del S1 con respecto al SO ó sistema fijo del manipulador. De esta forma, la matriz $A_{n}^{0}$, en la que $n$ es el grado de libertad, es aquella que representa la posición final del manipulador con respeto al sistema de referencia; esta matriz comúnmente es denominada $\mathrm{T}$ y se representa mediante las ecuaciones 1 y 2. [21], [22], [23]

$T=\prod_{i=1}^{n} A_{i}^{i-1}$

Con $n=6$. Es decir un sistema de 6 grados de libertad

$T=A_{n}^{0}=A_{1}^{0} \cdot A_{2}^{1} \cdot A_{3}^{2} \cdot A_{4}^{3} \cdot A_{5}^{4} \cdot A_{6}^{5}$

Cada matriz individual se evalúa mediante la ecuación (3):

$A_{i}^{i-1}=$

$[C \theta i ;-C \alpha \mathrm{i} * \mathrm{~S} \theta i ; \quad S \alpha \mathrm{i} * \mathrm{~S} \theta i ; \alpha \mathrm{i} * \mathrm{C} \theta i]$

$S \theta i ; C \alpha \mathrm{i} * \mathrm{C} \theta i ;-S \alpha \mathrm{i} * \mathrm{C} \theta i ; \alpha \mathrm{i} * \mathrm{~S} \theta i$

$\begin{array}{cccc}0 ; & S \alpha \mathrm{i} ; & C \alpha \mathrm{i} ; & d i \\ 0 ; & 0 ; & 0 ; & 1\end{array}$ 
Donde $C \theta i=\cos \theta i, C \alpha \mathrm{i}=\cos \alpha \mathrm{i}, \mathrm{S} \theta i=\operatorname{sen} \theta i \mathrm{y}$ $S \alpha \mathrm{i}=$ sen $\alpha \mathrm{i}$. Evaluando para cada $A_{n}^{0}$ : se obtienen las ecuaciones (4) al (9) para n igual a 1 hasta 6.

Se presenta que en todas las ecuaciones del (4) al (9), solo se tiene la variable $\theta i$ de cada articulación como era de esperarse, ya que se mencionó anteriormente que el manipulador es de tipo "6R".

Después de evaluar la ecuación (2) se obtiene la matriz T que es semejante a la expresión (10). De allí se extrae información referente a la rotación $\left(R_{3 \times 3}\right)$, la posición $\left(P_{3 \times 1}\right)$, la transformación de perspectiva $\left(f_{1 \times 3}\right)$ y el factor de escala global $\left(w_{1 \times 1}\right)$.

$\mathrm{T}=$\begin{tabular}{l|l}
$R_{3 \times 3}$ & $P_{3 \times 1}$ \\
$f_{1 \times 3}$ & $w_{1 \times 1}$
\end{tabular}

La cinemática directa termina, encontrando los valores de la matriz de orientación $\left(R_{3 \times 3}\right)$ del sistema de coordenadas i-ésimo establecido en el elemento $i$ con respecto al sistema de coordenadas de la base y el vector de posición $\left(P_{3 \times 1}\right)$ que apunta desde el origen del sistema de coordenadas de la base hasta el origen del sistema de coordenadas i-ésimo. En conclusión la cinemática directa permite determinar $R_{3 \times 3} \mathrm{y}$ $P_{3 x 1}=P x[i, j, k]+P y[i, j, k]+P z[i, j, k]$.

\subsection{Simulación:}

Haciendo uso del Matlab ${ }^{\circledR}$ se implementaron algunos segmentos de código para determinar las posiciones espaciales del efector final y poder comprobar los datos teóricos con los experimentales.

En la primera parte se realizó un programa para calcular las matrices de transformación individuales: $n=6 \%$ Definición del DOF

syms Q, alfa, a, d \%Parámetros DH

$\%$ Mat. De trasnf. individuales

for $i=1: n$

$A[i-1, i]=\left[\cos \left(Q^{*} i\right)-\quad \cos (a l f a * i)^{*} \sin \left(Q^{*} i\right)\right.$

$\sin (a l f a * i){ }^{*} \sin (Q * i)(a * i){ }^{*} \cos (Q * i)$;

$\sin (Q * i) \quad \cos (a l f a * i) * \cos (Q * i) \quad-\sin (a l f a * i) * \cos (Q * i)$

$(a * i)^{*} \sin (Q * i)$;

$0 \sin (a l f a * i) \cos (a l f a * i) d * i$

$\left.\begin{array}{lllllll}0 & 0 & 0 & 1\end{array}\right]$
End

Seguidamente se genera otro código para ingresar los parámetros $\mathrm{DH}$ y calcular el vector de posición $P_{3 \times 1}$ :

Q=input('Digite Los Valores Para Qi:')

alfa=input('Digite Los Valores Para ALFAi:')

a=input('Digite Los Valores Para ai:')

$\mathrm{d}=$ input('Digite Los Valores Para di:')

$\mathrm{T}=\mathrm{A} 01{ }^{*} \mathrm{~A} 12{ }^{*} \mathrm{~A} 23{ }^{*} \mathrm{~A} 34{ }^{*} \mathrm{~A} 45^{*} \mathrm{~A} 56$

$\mathrm{pX}(\mathrm{i}, \mathrm{j}, \mathrm{k})=\mathrm{T}(1,4)$

$\mathrm{pY}(\mathrm{i}, \mathrm{j}, \mathrm{k})=\mathrm{T}(2,4)$

$p Z(i, j, k)=T(3,4)$

Por último se realiza la simulación CAD del prototipo y se comparan las coordenadas en cada uno de las herramientas de software.

\subsection{Software de control:}

Desde el punto de vista del control del manipulador propuesto, se menciona que el módulo de entradas y salidas está basado en la tarjeta Mini Maestro 24-"Channel USB Servo Controller" y el módulo de control de motores es el "Pololu Jrk 12v 12 USB Motor Controller with Feedback" del "Fabricante Pololu Electronics". En ambos casos el proveedor entregó un software básico de control de cada tarjeta y este permite controlar por separado los actuadores del prototipo, generar secuencias de movimientos $y$ hasta pequeños programas que generen señales de control a cada actuador. Con el fin de integrar todo el control de hardware y personalizar su funcionamiento hacia comportamientos específicos se desarrolló una aplicación de prueba en Microsoft Visual C\# 2013(figura 11), la cual mediante el SDK (pololu-usb-sdk-140604) permitió integrar todo el control electrónico. 


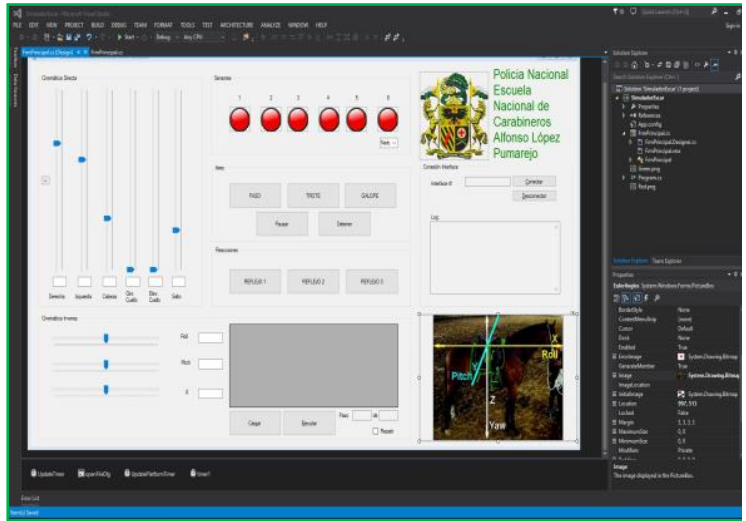

Figura. 11 Interfaz de prueba en MV C\# Fuente: Grupo de investigación UDEC-ESCAR

\section{Resultados}

Para ambos procedimientos de lectura (simulación y medida real) de posiciones se utilizan los mismos valores de entrada tanto en el Matlab como en la interfaz desarrollada; se introducen valores de ángulos que toman las articulaciones de acuerdo a la tabla 3 y se obtiene una posición en el espacio del extremo del robot manipulador; luego se compararon los resultados obtenidos y se observó que en la mayoría de los casos las medidas variaban significativamente en el orden de las decimas de centímetros especialmente para las tres primeras articulaciones.

Se pudo establecer en referencia a estas medidas que el error de posicionamiento se aproximaba al $38 \%$, además, que los actuadores respondían muy bien en ciertos trayectos del manipulador y aún cuando se tuvieron en cuenta valores preliminares de carga, cuando se llevan al final de carrera de cada actuador, vencer la inercia en ese punto hace que el motor trabaje lento y en algunos casos que no responda con las señales esperadas.

De igual manera se observó que los acoples mecánicos de todos los eslabones presentan una tolerancia promedio del $0,8 \%$, lo cual se debe tener presente a la hora de estimar el error en las medidas tomadas; de igual manera se estimaron errores de precisión y exactitud en la medida experimental, debidos a fallas humanas y de los instrumentos utilizados.

En general se logra el objetivo inicial de construir el prototipo y comprender de una manera apropiada los movimientos generados por el manipulador una vez dadas las secuencias o las ordenes de control, así como detectar posibles mejoras de forma que se realizaron en su momento y permitieron la implementación final del prototipo como se muestra en la figura 12.

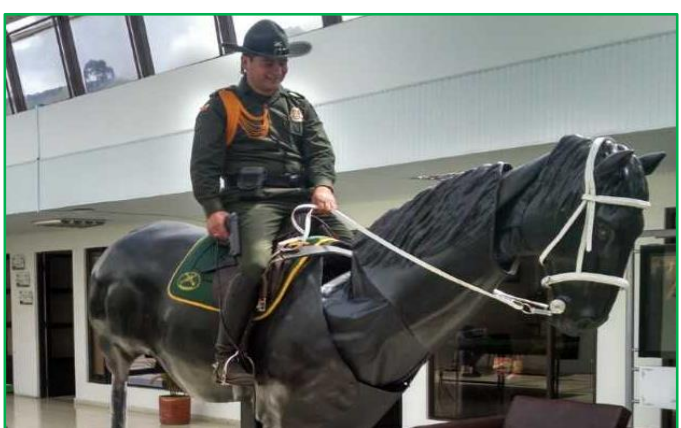

Figura. 12 Implementación final del robot Fuente: Grupo de investigación UDEC-ESCAR

Principalmente el sistema de simulación de tiro montado consta de una plataforma robótica equina que simula los movimientos propios del animal, un simulador gráfico que contiene las pruebas que debe ejecutar el jinete dentro de un entorno natural interactivo, un arma que permite dicha interacción, y un sistema electrónico de control o procesador central que comunica todas las partes.

El principal componente del simulador constituye una plataforma robótica con forma y apariencia de caballo, encargada de representar los movimientos característicos del equino: paso, trote y galope. Un requisito adicional para la construcción de este robot es la carga máxima que debe soportar, para este caso se estableció el umbral en $100 \mathrm{~kg}$, dejando un amplio rango para los jinetes que van a hacer uso del simulador, ya que pueden tener pesos variados.

Para lograr simular los movimientos equinos fue necesario realizar varias pruebas a caballos reales mediante sensores inerciales. Una vez se recolectaron esos datos se hizo un análisis exhaustivo de requerimientos de sensores y actuadores para su elección. Después de determinar los equipos y componentes electrónicos a utilizar, se procedió a realizar el 
diseño mecánico estructural para toda la plataforma, con base en las necesidades planteadas al principio. Luego de instalar las correspondientes partes sobre el esqueleto, se instaló la parte electrónica para establecer el control del caballo, integrándola con la mecánica y definiendo detalles finales como la apariencia del caballo lo más similar posible a la de uno real.

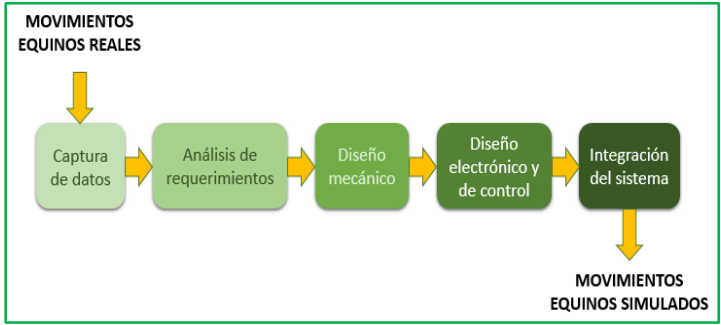

Fig. 13. Diagrama de bloques del proceso a seguir para simular los movimientos del caballo. Fuente: Grupo de investigación UDEC-ESCAR

\section{Discusión}

El análisis de movimientos mediante la metodología de Denavit y Hartenberg corresponde a un buen método matricial a la hora de establecer la cinemática directa de un manipulador, sin embargo se logró evidenciar en este estudio que el desarrollo analítico se complica cuando las restricciones del sistema permiten elevar el grado de libertad de un manipulador; como alternativa se debe migrar a herramientas computacionales y aún así los recursos de maquina deben ser suficientes para lograr la demanda de recursos, cálculos y señales gráficas. Trabajos futuros se centrarán en otros métodos de análisis de movimientos, análisis dinámico, de trayectorias y de sistemas de control.

\section{Conclusiones}

Se diseñó y construyó el prototipo de robot equino con seis grados de libertad para fines académicos en la ESCAR.

Una vez terminada la implementación, el mismo prototipo puede ser utilizado para realizar otras prácticas como simulador equino e inclusive puede ser reprogramado y servir como herramienta en la detección de lesiones óseas equinas.
Debido a su modularidad estructural, de hardware y de software, puede servir como referente para el análisis de mecanismos equinos, además, al ser escalable permite la adición de sensores y otros actuadores que pueden dar origen al diseño de controladores electrónicos específicos.

Gracias al uso de herramientas CAD se logró el diseño geométrico del robot equino, lo cual permitió la evaluación y consideración de diversos aspectos del mismo antes de la construcción e implementación, reduciendo así tiempos de fabricación menos cantidad de prototipos físicos, lo que se traduce en menos costos en el desarrollo del sistema simulador de tiro.

Se logró el análisis de movimientos del robot equino gracias a herramientas como Matlab ${ }^{\circledR}$ y kinovea $\mathbb{R}$ que permitieron reducir en gran medida el tiempo de diseño del prototipo y contribuyeron para la realización de simulaciones y variaciones de los parámetros variables dentro del manipulador hasta llegar a proporcionar una buena comprensión de los movimientos del prototipo desarrollado.

\section{References}

[1] Rachid Manseur, "Software -Aided Robotics Education and Design", Electrical and Computer Engineering Dept. State University of New York at Oswego, Oswego, NY, USA. 2016

[2] Henry W. Stone, Arthur C. Sanderson, and Charles P. Neuman," arm signature identification", Department of Electrical and Computer Engineering and Robotics Institute, Carnegie-Mellon University.1986.

[3] Asghar khan, Wang Li Quan, "Forward Kinematic Modeling and Analysis of 6-DOF Underwater Manipulator", School of Mechanical and Electrical Engineering, Harbin Engineering University Harbin, China. 2015.

[4] Ondrej Hackl, Peter Drgoiia2, Marek Paskala3, "Simulation Model of Adjustable Arm Using DenavitHartenberg Parameters", Department of 
electronics and mechatronics Faculty of Electrical Engineering University of Zilina, Slovakia. 2014.

[5] Muhammad Rameezl and Dr. Liaquat Ali Khan, "Modeling and Dynamic Analysis of the Biped Robot", Department of Mechatronics Engineering, Air University Islamabad, Pakistan, 2014.

[6] Xiaolun Shi Department of Systems Engineering, The Chinese University of Hong Kong R.G. Fenton, "A Complete and General Solution to the Forward Kinematics Problem of Platform-Type Robotic Manipulators", Department of Mechanical Engineering The University of Toronto. Toronto.1994.

[7] A.J. van den Bogert, A.A.H.J. Sauren* and H.C. Schamhardt,"Analysis Of Locomotion In The Horse Using Computer Aided Engineering Software" Department of Veterinary Anatomy, University of Utrecht, The Netherlands *Department of Mechanical Engineering , Eindhoven University of Technology The Netherlands, Netherlands.1988.

[8] C. M. Cordova-Villarreal, J. C. MontesdeocaContreras, R. S. Avila-Campoverde, "Study and Design of Biped Robot ofTen DOF for Stability Analysis in Walking Process", Carrera de Ingenieria Electr6nica, Universidad Politecnica Salesiana, Cuenca-Ecuador, 2015.

[9] Jungsan Cho, Sangdeok Park and Kabil Kim,"Design of mechanical stiffness switch for hydraulic quadruped robot legs inspired by equine distal forelimb", Electronics Letters, Vol. 51 No. 1 pp. 33-35. 2015

[10] Mohammad Afif Ayob, Wan Nurshazwani Wan Zakaria, "Forward Kinematics Analysis of a 5-Axis RV-2AJ Robot Manipulator", Departmentof Mechatronic and Robotic Engineering, Faculty of Electrical and Electronic Engineering, UniversitiTun Hussein Onn Malaysia, 86400 Parit Raja, BatuPahat, Malaysia. 2014.

[11] Shuxiang Guo1,2, Yuehui Ji1 Lin Bi1, Xu Ma1 and Yunliang Wang1," A Kinematic Modeling of an Amphibious Spherical Robot System", Tianjin
Key Laboratory for Control Theory \& Applications in Complicated Systems and Biomedical Robot Laboratory, Systems Eng. Depart Kagawa University, Tianjin University of Technology. Tianjin, China, 2014.

[12] Zafra, S., Martínez, J. y Vergel, M. Indicadores para evaluar la pertinencia social en la oferta académica de programas. Revista Logos, Ciencia \& Tecnología. 6 (1): 142-155, 2014. [Fecha de consulta: 7 de enero de 2019]. Disponible en: https://www.redalyc.org/articulo.oa?id=5177515 50012

[13] Santichai Fueanggan, Somchart Chokchaitam, "Dynamics and Kinematics Simulation for Robots", Dept. Electrical and Computer Engineering Thammasat University, Pathumthani, Thailand. 2009.

[14] Jack C. K. Chou George Baciu H. K. Kesavan, "Graph-Theoretic Models For Simulating Robot Manipulators", Department of Systems Design Engineering, University of Waterloo, Waterloo, Ontario, Canada N2L 3G1.1987.

[15] Xinjie WANG1, Xuedong CHEN 1,2 Wenchuan JIA 2, Yi SUN 2 and Huayan PU 2, "Forward Kinematics Analysis and 3-Dimmision Gait Simulation of a MiniQuad Walking Robot", Wuhan, 430074, P. R. China. 2007.

[16] Torres F, Pomares J, Gil P, Puente S y Aracil R, "Robots y sistemas sensoriales", Pearson Educación S.A, Madrid, España.2002.

[17] Lei Li and Qidan Zhu Liyan Xu," Solution for Forward Kinematics of 6-DOF Parallel Robot Based on Particle Swarm Optimization", College of Automation College of Science, Harbin Engineering University Harbin Engineering University, Harbin,China. 2007.

[18] Sung-Hua Chen, Chin-I Huang, and Li-Chen Fu, "Applying a Nonlinear Observer to Solve Forward Kinematics of a Stewart Platform", Fellow, IEEE, San Antonio, Texas, USA, 2008.

[19] Luc Rolland, Rohitash Chandra "Forward Kinematics of the 6-6 general Parallel 
Manipulator Using Real Coded Genetic

Algorithms", Mechanical Engineering dept.

Kalkanli, Turkish Republic of North Cyprus.

Singapore, 14-17, 2009

[20] Barrientos A, Peñín L, Balaguer C y Aracil R," Fundamentos de robótica", McGraw Hill, Interamericana de España. C.A, Madrid. España. 2007.

[21] Rong Xuewen Li Yibin Song Rui, "Kinematic Analysis of A Shotcreting Robot", School of Control Science and Engineering Shandong University 73, Jingshi Road, Jinan, 250031, China. 2010.

[22] Wang Pin, Lu Hong, Wang Shi'en, "New Method for Input-output Equation of Spherical Four-bar Mechanism", Key Lab. of Advanced Manufacturing and Automation Technology Ludong University Yantai, Shandong Province, China. 2011.

[23] Hang Wang, Hanghang Qi, Minghui Xu, Yanhua Tang, Jiantao Yao, Xuedong Yan, Ming Li, "Research on the Relationship between Classic Denavit-Hartenberg and Modified DenavitHartenberg", china, 2014. 